\title{
"Calibration of aerodynamic roughness over the Tibetan Plateau with Ensemble Kalman Filter analysed heat flux" published in Hydrol. Earth Syst. Sci., 16, 4291-4302, 2012
}

\author{
J. H. Lee ${ }^{1,2}$, J. Timmermans ${ }^{2}$, Z. Su ${ }^{2}$, and M. Mancini ${ }^{1}$ \\ ${ }^{1}$ Politecnico di Milano, Piazza Leonardo da Vinci 32, Milano, Italy \\ ${ }^{2}$ ITC, Geo-Information Science and Earth Observation of the University of Twente, Enschede, The Netherlands \\ Correspondence to: M. Mancini (marco.mancini@ polimi.it)
}

We would like to inform you that there is a mistake in the following sentence on p. 4296 (right column):

Because of following relationships $A^{\mathrm{a}}=A^{\mathrm{f}}+K(D-$ $\left.\mathbf{H} A^{\mathrm{f}}\right) \cong A^{\mathrm{f}} K \mathbf{H} A^{\mathrm{f}}$ and $D=0, P^{\mathrm{f}} \mathbf{H}^{T} K^{T}=K \mathbf{H} P^{\mathrm{f}}$, then error covariance in Eq. (3-3) is rearranged as followed (Sakov et al., 2008).

The correct sentence should read:

Because of following relationships $A^{\mathrm{a}}=A^{\mathrm{f}}+K\left(D-\mathbf{H} A^{\mathrm{f}}\right)$, $D=0$, and $P^{\mathrm{f}} H^{T} K^{T}=K \mathbf{H} P^{\mathrm{f}}$, then error covariance in Eq. (3-3) is rearranged as followed. 